\title{
Estudo comparativo do emprego da aprotinina em baixas doses $X$ placebo, durante a circulação extracorpórea
}

\author{
José Carlos D. V. PONTES*, Maria de Fátima C. MATOS*, Carlos Geraldo S. MEDEIROS*, \\ Arino F. SILVA*, João J. DUARTE*, Neimar GARDENAL*, Marcos Douglas Z. VIOLA*
}

RBCCV 44205-571

\begin{abstract}
Pontes J C D V, Matos M F C, Medeiros C G S, Silva A F, Duarte J J, Gardenal N, Viola M D Z - Estudo comparativo do emprego da aprotinina em baixas doses $X$ placebo, durante a circulação extracorpórea. Rev Bras Cir Cardiovasc 2002; 17(1): 47-53.
\end{abstract}

RESUMO: Fundamentos: A aprotinina, um antifibrinolítico de natureza protéica, tem sido proposta pela literatura, no intuito de minimizar os efeitos adversos da circulação extracorpórea ao sistema fibrinolítico, permitindo assim uma hemostasia mais adequada.

Objetivo: Estudar comparativamente o efeito da utilização profilática de baixas doses de aprotinina em pacientes submetidos à circulação extracorpórea.

Método: Dezessete pacientes portadores de valvopatia mitral submetidos a correção cirúrgica utilizandose circulação extracorpórea foram aleatoriamente divididos em dois grupos: I (controle) - 9 pacientes nos quais foi administrado placebo no perfusato e a cada hora de CEC; II (aprotinina) - 8 pacientes nos quais foram administrados $30000 \mathrm{KIU} / \mathrm{kg}$ e $7500 \mathrm{KIU} / \mathrm{kg}$ de aprotinina adicionados ao volume do perfusato a cada hora de CEC. O volume total de sangramento pós-operatório nas primeiras 24 horas foi monitorado, assim como amostras sangüíneas foram colhidas após indução anestésica e após a neutralização da heparina com sulfato de protamina para que os seguintes parâmetros fossem avaliados: atividade de protrombina (AP), tempo de tromboplastina parcial ativado (TTPA), tempo de trombina (TT), dosagem de fibrinogênio, concentração de antitrombina III (ATIII), tempo de lise da euglobulina (TLE) e dosagem do dímero -D (DDi).

Resultados: $O$ volume médio total de sangramento nas primeiras 24 horas no grupo controle foi $690,67 \pm 377,12$, enquanto que no grupo da aprotinina em baixas doses foi de $248,75 \pm 103,13$ ( $p=0,0017$ ). No quadro abaixo estão apresentados os resultados obtidos a partir das análises das amostras sangüíneas pré e pós-operatórias comparativas dos grupos controle (I) e aprotinina (II).

\begin{tabular}{lccc}
\hline Exame & Período & Grupo I & Grupo II \\
AP & pré & $81,2 \pm 13,3$ & $83,6+14,92$ \\
Vr-70-130Seg & pós & $54,5 \pm 11,0$ & $61,3+12,25$ \\
TIPA & pré & $34,1 \pm 8,1$ & $34,9+6,39$ \\
Vr-22-35Seg & pós & $40,6 \pm 7,1$ & $44,9+8,06$ \\
TT & pré & $15,4 \pm 1,1$ & $15,9+2,38$ \\
Vr-14-21Seg & pós & $17,5 \pm 1,1$ & $17,7+2,26$ \\
Fibrinog & pré & $3,04 \pm 0,84$ & $3,16+1,57$ \\
Vr-1,8-3,5g/dl & pós & $1,90 \pm 0,36$ & $2,28+0,46$ \\
ATIII & pré & $21,8+2,9$ & $21,7+3,09$ \\
Vr-22-39mg/dl & pós & $19,1 \pm 2,9$ & $20,7+2,93$ \\
TLE & pré & $160 \pm 25,30$ & $158,7+3,30$ \\
Vr-120-300min & pós & $127 \pm 61,69$ & $146,2+32,7$ \\
\hline
\end{tabular}

Conclusão: A utilização da aprotinina, em baixa dose, foi eficaz na redução do sangramento, assim na diminuição da fibrinólise.

DESCRITORES: Aprotinina, uso terapêutico. Circulação extracorpórea, efeitos adversos.

\footnotetext{
Trabalho realizado no Serviço de Cirurgia Cardiotorácica do Núcleo do Hospital Universitário da Universidade Federal de Mato Grosso do Sul. Campo Grande, MS, Brasil.

Recebido para publicação em janeiro de 2001.

* Da Disciplina de Cirurgia Cardiotorácica da Universidade Federal de Mato Grosso do Sul.

Endereço para correspondência: José Carlos D. Pontes. Rua Afonso Pena, 3088, apto 502. CEP 79100-000. Campo Grande, MS, Brasil. Tel: (67) 9981-1670.784-6180.e-mail: jcarlosdorsa@uol.com.br
} 
Pontes J C D V, Matos M F C, Medeiros C G S, Silva A F, Duarte J J, Gardenal N, Viola M D Z - Estudo comparativo do emprego da aprotinina em baixas doses X placebo, durante a circulação extracorpórea. Rev Bras Cir Cardiovasc 2002; 17(1): 47-53.

\section{INTRODUÇÃO}

A cirurgia cardíaca teve um espetacular desenvolvimento nos últimos anos, com as possibilidades oferecidas pela circulação extracorpórea (CEC), permitindo a correção das mais diversas cardiopatias.

A circulação extracorpórea interfere em toda a fisiologia do organismo, estimulando, dessa forma, o estudo contínuo dos seus efeitos e complicações, no intuito de que as adversidades do método possam ser contornadas ou minimizadas.

O contato do sangue com a superfície não endotelizada dos circuitos da CEC ativa o fator XII, desencadeando a ativação da via extrínseca da cascata da coagulação, independentemente da heparinização. A produção de plasmina também é estimulada em função da ativação do fator XII.

Ainda durante a circulação extracorpórea, devido à hemodiluição, à hipotermia, ao trauma dos elementos figurados do sangue e à liberação de substâncias vasoativas, observa-se alterações nas plaquetas, nas proteínas relacionadas à coagulação e no sistema fibrinolítico, conforme descreveram HORROW (1), ELLISON \& JOBES ${ }^{(2)} \mathrm{e}$ KUCUK et al. (3).

A fibrinólise, como fator causal de sangramento pós-CEC, está consensuada na literatura pertinente, conforme relatado por BIDSTRUP ${ }^{(4)}$. A tentativa de resolução do problema na esfera farmacológica remonta à década de sessenta, quanto TICE et al. (5), em 1964, propuseram pioneiramente a utilização da aprotinina na circulação extracorpórea (CEC).

A aprotinina é um antifibrionolítico de natureza protéica que atua sobre as proteases séricas, inibindo a tripsina, quimiotripsina, plasmina, t-PA e a calicreína. A aprotinina também inibe a agregação plaquetária induzida pelo colágeno, epinefrina, ADP, ácido aracdônico e trombina in vitro.

A utilização desse inibidor de serino proteinases, de maneira profilática, durante a CEC, tem-se mostrado capaz de reduzir o sangramento e a necessidade de transfusão sangüínea, como observado por VAN OEVEREN et al. ${ }^{(6)}$.

ROYSTON et al. (7) e BIDSTRUP et al. (8) padronizaram a utilização de altas doses de aprotinina após indução anestésica, demonstrando a redução de perdas sangüíneas, assim como demonstraram seu efeito na hemostasia. Essa observação foi confirmada por vários autores, conforme relatos de LEMINER et al.(9), GOLDSTEIN et al. (10), COLMAN (11) e HERYNKOPF et al. (12).

Analisando-se estudos mais recentes, HAVEL et al. (13), em 1994, LIU et al. (14), em 1995,
MASTROROBERTO et al. (15), em 1995, observaram efeitos satisfatórios na prevenção da fibrinólise com baixas dosagens de aprotinina.

Considerando-se a possibilidade de resposta adversa às altas dosagens dessa droga, uma vez sendo proteína animal, além do seu alto custo, acredita-se ser de valia o estudo da utilização de baixas dosagens de aprotinina com finalidade de promover diminuição das perdas sangüíneas pósCEC, evitando-se a utilização excessiva dos hemoderivados.

A presente investigação tem por objetivo estudar o efeito antifibrinolítico da aprotinina em baixas doses, assim como sua influência sobre o volume total de sangramento no pós-operatório imediato de pacientes submetidos à circulação extracorpórea.

\section{CASUÍSTICA E MÉTODOS}

Dezessete pacientes portadores de insuficiência mitral foram selecionados para a realização deste estudo prospectivo.

Foram excluídos do estudo os pacientes portadores de insuficiência renal, diabete melito, doença pulmonar obstrutiva crônica (DPOC), doença hematológica, hepática ou digestiva. Foram excluídos, também, os casos de reoperação e os pacientes que faziam uso prévio de anti-inflamatórios não hormonais incluindo-se o ácido acetil salicílico.

Os pacientes foram, consecutivamente, submetidos à circulação extracorpórea para substituição valvar mitral. Todos foram operados pela mesma equipe da Disciplina de Cirurgia Cardiotorácica do Hospital Universitário da Universidade Federal de Mato Grosso do Sul (UFMS).

Os 17 pacientes foram aleatoriamente divididos em dois grupos:

Grupo I: (controle) 9 pacientes nos quais, após a indução anestésica, foi administrado placebo em veia central e no perfusato a cada hora de CEC.

Grupo II: (aprotinina) 8 pacientes submetidos à baixa dose de aprotinina. Foram administrados 30000 $\mathrm{KIU} / \mathrm{kg}$, após a indução anestésica em veia central e adicionados ao volume do perfusato $7500 \mathrm{KIU} / \mathrm{kg}$ a cada hora de CEC.

A antibioticoprofilaxia foi realizada, em todos os pacientes, de acordo com as normas da Comissão de Controle de Infecção Hospitalar do Hospital Universitário da UFMS, administrando-se $2 \mathrm{~g}$ de cefazolina, uma hora antes da operação, e, em seguida, $1 \mathrm{~g} \mathrm{EV} \mathrm{6/6} \mathrm{h,} \mathrm{durante} 72$ horas. 
Pontes J C D V, Matos M F C, Medeiros C G S, Silva A F, Duarte J J, Gardenal N, Viola M D Z - Estudo comparativo do emprego da aprotinina em baixas doses X placebo, durante a circulação extracorpórea. Rev Bras Cir Cardiovasc 2002; 17(1): 47-53.

A técnica anestésica empregada seguiu a rotina do Serviço, indução anestésica: fentanil, 5 e $10 \mathrm{mcg} /$ $\mathrm{kg}$; etomidato, 0,2 a 0,3 $\mathrm{mg} / \mathrm{kg}$ e pancurônio, na dose de 0,1 a $0,2 \mathrm{mg} / \mathrm{kg}$. Na manutenção, foram administrados, a cada hora de procedimento, fentanil $5 \mathrm{mcg} / \mathrm{kg}$ e pancurônio $1 \mathrm{mg} / \mathrm{kg}$. Nesse período, também foi associado o isoflurano, como agente anestésico inalatório, na dose de $0,5 \%$ a $1,5 \%$ de concentração alveolar. A ventilação pulmonar foi mantida através de respirador automático de pressão positiva intermitente com sistema semi-fechado com absorvente de $\mathrm{CO}_{2}$.

A abordagem cirúrgica em todos os pacientes foi através de esternotomia mediana.

\section{Técnica de Circulação Extracorpórea}

Foram utilizados bomba de rolete na linha arterial e oxigenadores de membranas de fibras ocas com filtro de linha arterial (Braile Biomédica - São José do Rio Preto - SP) e com perfusão de $1500 \mathrm{ml}$ aproximadamente. As perfusões foram realizadas em hipotermia a $27^{\circ} \mathrm{C}$ e hemodiluição total e o método de proteção miocárdica foi cardioplegia fria (4 a 8 graus ${ }^{\circ} \mathrm{C}$ ) cristalóide St. Thomas anterógrada a cada 20 minutos após o pinçamento aórtico. Todo o material artificial descartável empregado na CEC não possuía revestimento de heparina (circuito não heparinizado). Em todos os pacientes a CEC foi instalada com canulação da aorta ascendente e drenagem venosa através de canulação das cavas em separado, após heparinização sistêmica com $4 \mathrm{mg} / \mathrm{kg}$, repetida de acordo com o TCA (tempo de coagulação ativado), com o objetivo de mantê-lo acima de 450 segundos. A neutralização da heparina foi realizada, em todos os casos, com infusão de protamina na dose de 1:1, diluída em soro glicosado $5 \%$.

\section{Cuidados Pós-Operatórios}

Ao término da operação, os pacientes ainda sob anestesia geral e em normotermia foram encaminhados à Recuperação Cardíaca Pós-operatória, intubados e mantidos sob ventilação mecânica com respiradores microprocessados ciclados a volume, com $\mathrm{FiO}_{2}$ necessária para manter a $\mathrm{PaO}_{2}$ acima de $100 \mathrm{mmHg}$. A reposição de volume no pós-operatório foi feita com soluções cristalóides acrescidas de eletrólitos, no sentido de manter bons parâmetros hemodinâmicos, equilíbrio hidroeletrolíticos e ácidobásico. Drogas vasoativas foram usadas quando necessário, para controle e manutenção da função hemodinâmica. Não foram prescritos anti-agregantes plaquetários, ou qualquer tipo de anti-inflamatório.

\section{Parâmetros analisados:}

A) Sangramento - Foram registrados os volumes totais de sangramento pós-operatório nas primeiras 24 horas.

B) Exames laboratoriais:

a. Testes globais da coagulação: atividade de protrombina $(\mathrm{AP})$, tempo de tromboplastina parcial ativado (TTPA), tempo de trombina (TT) e dosagem de fibrinogênio por turbo-densitometria (Behring Fibrintimer).

b. Determinação da concentração da antitrombina III (ATIII) por turbidimetria (Behring TurbiTime System). Método analítico onde a turbidez produzida pelos complexos antígenoanticorpo é medida fotometricamente.

c. Tempo de lise da euglobina (TLE) pelo método de BUCKELL.

d. Dosagem do dímero -D (DDi) por método de aglutinação em látex utilizando o kit da Organon Teknika.

\section{Coleta das Amostras Sangüíneas}

Foram colhidas amostras sangüíneas em dois tempos:

1 - Após indução anestésica na sala de operação

2 - Após a neutralização da heparina com sulfato de protamina

Para a realização dos testes de hemostasia, foram coletados $10 \mathrm{ml}$ de sangue da artéria radial utilizando-se seringas e tubos de plástico com citrato de sódio a 3,8\% na proporção de uma parte de anticoagulante para nove partes de sangue total. Estas amostras foram centrifugadas a 3000 rpm durante 15 minutos para obtenção do plasma pobre em plaquetas (PPP), o qual foi dividido em alíquotas de 500 microlitros, congelados rapidamente em nitrogênio líqüido e estocado em "freezer" a $-70^{\circ} \mathrm{C}$, para ensaios posteriores.

\section{Método Estatístico}

Para a análise estatística dos resultados, foram aplicados os seguintes testes: "t" de Student e MannWhitney. 
Pontes J C D V, Matos M F C, Medeiros C G S, Silva A F, Duarte J J, Gardenal N, Viola M D Z - Estudo comparativo do emprego da aprotinina em baixas doses X placebo, durante a circulação extracorpórea. Rev Bras Cir Cardiovasc 2002; 17(1): 47-53.

\section{RESULTADOS}

A análise dos grupos quanto ao sexo mostrou, no Grupo I, $3(33,3 \%)$ pacientes do sexo masculino e $6(66,7 \%)$ do sexo feminino. No Grupo II, 4 (50\%) pacientes do sexo masculino e $4(50 \%)$ pacientes eram do sexo feminino.

A idade, no Grupo I, variou de 18 a 70 anos (média: $56 \pm 11,7$ anos) e no Grupo II, de 22 a 75 anos (média: 62,0 \pm 9,4 anos).

O peso, no Grupo I, foi de 56 a $87 \mathrm{~kg}$ (média: $64,2 \pm 8,2 \mathrm{~kg}$ ) e, no Grupo II, de 42 a $92 \mathrm{~kg}$ (média: $57,5 \pm 11,3 \mathrm{~kg}$ ).

Quanto à altura, variou, no Grupo I, de 1,45 a 1,82m (média: 1,69 $\pm 1,2 \mathrm{~m}$ ) e, no Grupo II, de 1,50 a 1,84 $\mathrm{m}$ (média: $1,72 \pm 2,1 \mathrm{~m}$ ).

O tempo total de operação variou, no Grupo I, de 180 a 350 min (média: $284,2 \pm 45,3 \mathrm{~min}$ ) e, no Grupo II, de 190 a $360 \mathrm{~min}$ (média: 286,3 \pm 38,0 min).

O tempo de CEC foi, no Grupo I, de 45 a 105 min (média: $65 \pm 15,2 \mathrm{~min}$ ) e, no Grupo II, de 45 a $110 \mathrm{~min}$ (média: 66,3 \pm 13,5 min).

O tempo de anóxia, no Grupo I, variou de 45 a 75 min (média: $55 \pm 8,2 \mathrm{~min}$ ) e, no Grupo II, de 38 a 72 min (média: 50,2 $\pm 7,1 \mathrm{~min}$ ).

No Gráfico 1 estão apresentados os valores médios do volume de sangue perdido nas primeiras 24 horas de pós-operatório, demonstrando redução estatisticamente significativa do volume de sangue drenado no grupo onde se aplicou a aprotinina.

Nas Tabelas 1 e 2 estão demonstrados os resultados dos testes AP, TTPA, TT, dosagem salto de febrinogênio, AT III, tempo de lise da euglobilina (TLE) e dosagem de dimero-D (DDi), respectivamente para os grupos controle e tratado com aprotinina.

\section{COMENTÁRIOS}

Considerando-se o volume de sangramento no período de pós-operatório, neste ensaio, observouse redução estatisticamente significativa do mesmo quando se comparou ao grupo controle, conforme está demonstrado no Gráfico 1.

DIETRICH et al. (16), estudando pacientes submetidos à revascularização primária, encontraram sangramento de $738 \mathrm{ml}$ no grupo que recebeu a aprotinina, comparado com $1431 \mathrm{ml}$ no grupo controle, que recebeu placebo.

GOSGROVE et al. (17) estudaram a influência da dose de aprotinina na eficiência da profilaxia, em pacientes submetidos à reoperação para revascularização do miocárdio. Um grupo de pacientes seguiu o protocolo de altas doses de Hammersmith; outro grupo recebeu metade da dose de Hammersmith e o terceiro grupo recebeu placebo. Os grupos que receberam aprotinina (dose total ou meia dose) apresentaram perda sangüínea semelhante e menor que o grupo que recebeu o placebo. A meia dose de aprotinina nos pacientes estudados foi tão eficaz quanto a dose total de Hammersmith.

A meia dose de Hammersmith ou "dose baixa" consiste de: 1.000.000 Ul administrados logo após a intubação traqueal; $250.000 \mathrm{Ul} / \mathrm{hora}$ em infusão venosa e $1.000 .000 \mathrm{UI}$ colocados no perfusato, antes do início da perfusão.

$\mathrm{Na}$ presente investigação, onde foram administrados $30000 \mathrm{KIU} / \mathrm{kg}$ após indução anestésica e no perfusato $7500 \mathrm{KIU} / \mathrm{kg}$ a cada hora de CEC, obser-

\section{GRÁFICO 1}

VOLUME MÉDIO DE SANGRAMENTO EM MILILITROS NAS 24 HORAS DE PÓS-OPERATÓRIO EM AMBOS GRUPOS

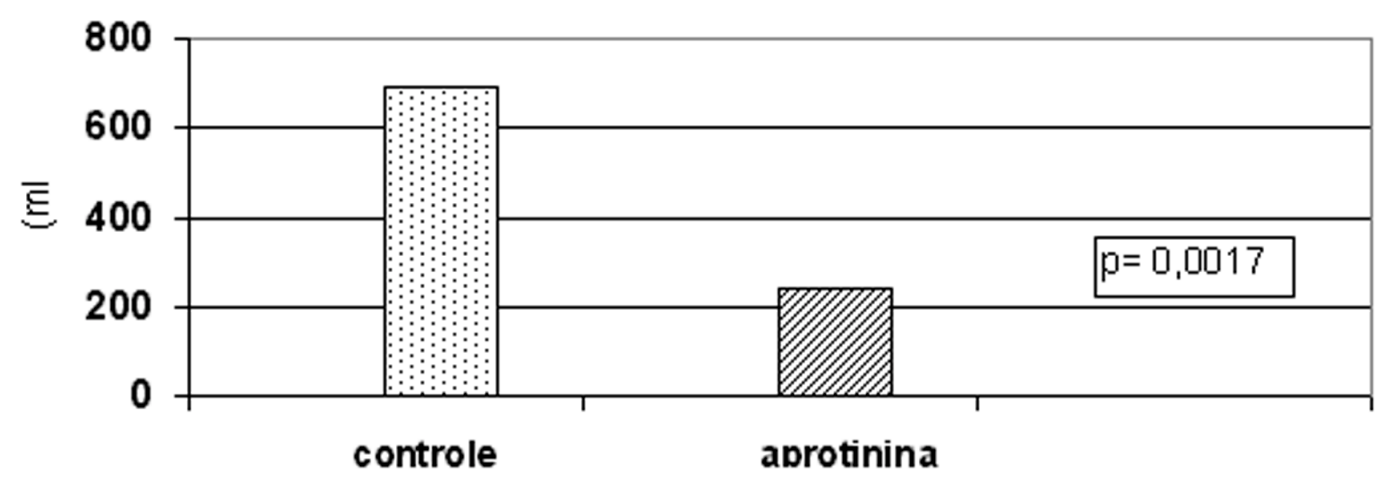


Pontes J C D V, Matos M F C, Medeiros C G S, Silva A F, Duarte J J, Gardenal N, Viola M D Z - Estudo comparativo do emprego da aprotinina em baixas doses X placebo, durante a circulação extracorpórea. Rev Bras Cir Cardiovasc 2002; 17(1): 47-53.

vou-se que, em relação aos testes de coagulação AP, TTPA, TT e às dosagens de AT III fibrinogênio, cujos valores individuais estão demonstrados nas Tabelas 1 e 2, somente a dosagem de fibrinogênio apresentou diferença significativa $(p=0,0166)$, entre o grupo que recebeu aprotinina e o controle. Para os outros testes citados, não houve diferença estatisticamente significativa entre esses dois grupos.

LIU et al. (18), estudando doses baixas de aprotinina, demonstraram redução de $40 \%$ no sangramento e $85 \%$ na necessidade de transfusão de hemácias, em comparação ao grupo controle.

$\mathrm{Na}$ tentativa de estabelecer a mínima dose eficaz, HARDY et al. administraram aprotinina em um grupo de 41 pacientes, na dose de 200.000 UI EV, seguida da infusão de 100.000 UI/hora durante oito horas, sem adição ao perfusato, não havendo diferença nos níveis de sangramento e outros parâmetros analisados.

No que se refere aos exames que avaliam a fibrinólise, observou-se, ainda, neste estudo, con- forme demonstrado nas Tabelas 1 e 2 , altos níveis de DDi no pós-operatório dos dois grupos. Houve, contudo, diferença estatisticamente significativa no grupo que recebeu a droga $(p=0,0065)$, indicando que a aprotinina, mesmo em baixas doses, inibiu a fibrinólise. A menor redução na concentração de fibrinogênio, no grupo que recebeu aprotinina, também pode estar relacionada a menor degradação deste fator, pela menor ativação da fibrinólise em relação ao grupo controle. Outro teste, o TLE, também evidenciou esta menor fibrinólise no grupo tratado em relação ao controle, entretanto, as diferenças não foram significativas $(p>0,05)$.

Embora os níveis de DDi no pós-operatório de pacientes tratados com altas doses de aprotinina sejam muito menores (BIDSTRUP et al. ${ }^{(4)}$ ), há que se considerar o elevado custo dessa droga, o que leva à impossibilidade de sua utilização rotineira na maioria das instituições públicas em nosso meio. Assim sendo, a utilização de baixa dose pode ser uma alternativa satisfatória, considerando-se a redução do sangramento observada neste trabalho.

TABELA 1

\begin{tabular}{|c|c|c|c|c|c|c|c|c|c|c|c|}
\hline Exame & & 1 & 2 & 3 & 4 & 5 & 6 & 7 & 8 & 9 & média + SD \\
\hline AP & pré & 83,3 & 79,3 & 72,4 & 94,3 & 100 & 70,9 & 55,2 & 95,2 & 80,0 & $81,2+13,3$ \\
\hline /r-70-130Seg & pós & 51,2 & 55,2 & 37,8 & 59,5 & 60,6 & 49,2 & 39,2 & 63,2 & 74,6 & $54,5+11,0$ \\
\hline TPA & pré & 28,4 & 31,9 & 34,1 & 30,3 & 28,0 & 42,6 & 53,6 & 30,4 & 28,0 & $34,1+8,1$ \\
\hline /r-22-35Seg & pós & 35,7 & 34,7 & 40,4 & 49,5 & 38,5 & 48,9 & 49,9 & 39,4 & 28,2 & $40,6+7,1$ \\
\hline $\mathrm{T}$ & pré & 14,0 & 15,1 & 15,5 & 15,2 & 15,7 & 17,6 & 14,1 & 16,7 & 14,7 & $15,4+1,1$ \\
\hline /r-14-21Seg & pós & 17,3 & 15,2 & 16,1 & 18,1 & 18,7 & 17,9 & 18,1 & 18,1 & 18,0 & $17,5+1,1$ \\
\hline ibrinog & pré & 3,56 & 3,56 & 2,29 & 2,37 & 2,18 & 3,58 & 1,81 & 2,79 & 3,1 & $3,04+0,84$ \\
\hline$/ \mathrm{r}-1,8-3,5 \mathrm{~g} / \mathrm{dl}$ & pós & 1,78 & 2,37 & 1,68 & 1,49 & 1,15 & 2,37 & 1,49 & 1,49 & 2,24 & $1,90+0,36$ \\
\hline ATIII & pré & 23,6 & 22,0 & 18,3 & 20,0 & 25,1 & 21,9 & 27,2 & 18,3 & 20,0 & $21,8+2,9$ \\
\hline$/ \mathrm{r}-22-39 \mathrm{mg} / \mathrm{dl}$ & pós & 17,7 & 20,8 & 17,7 & 17,5 & 21,1 & 19,5 & 25,7 & 15,4 & 16,8 & $19,1+2,9$ \\
\hline LE & pré & 180 & 180 & 180 & 100 & 180 & 100 & 160 & 180 & 180 & $160+25,30$ \\
\hline $\mathrm{r}-120-300 \mathrm{~min}$ & pós & 20 & 105 & 180 & 20 & 180 & 20 & 180 & 60 & 180 & $127+61,69$ \\
\hline $\mathrm{Di}^{*}$ & pré & $<500$ & $500-1000$ & $500-1000$ & 2000 & $<500$ & 2000 & $<500$ & 2000 & $500-1000$ & \\
\hline & pós & 2000 & 4000 & 2000 & 4000 & 2000 & 4000 & 4000 & 4000 & 1000 & \\
\hline
\end{tabular}

TABELA 2

\begin{tabular}{|c|c|c|c|c|c|c|c|c|c|c|}
\hline Exame & & 1 & 2 & 3 & 4 & 5 & 6 & 7 & 8 & média + SD \\
\hline AP & pré & 73,5 & 88,4 & 79,3 & 62,8 & 64,5 & 101,0 & 100 & 100 & $83,6 \pm 14,92$ \\
\hline Vr-70-130Seg & pós & 61,3 & 74,6 & 49,2 & 45,0 & 76,3 & 71,9 & 45,6 & 66,6 & $61,3 \pm 12,25$ \\
\hline TTPA & pré & 38,8 & 31,1 & 29,3 & 47,9 & 30,7 & 41,0 & 30,7 & 30,0 & $34,9 \pm 6,39$ \\
\hline Vr-22-35Seg & pós & 43,2 & 33,8 & 43,3 & 60,9 & 38,1 & 40,0 & 52,2 & 48,3 & $44,9 \pm 80,06$ \\
\hline TT & pré & 17,1 & 14,8 & 14,2 & 14,5 & 11,9 & 17,7 & 19,7 & 17,9 & $15,9 \pm 2,38$ \\
\hline Vr-14-21Seg & pós & 19,4 & 15,4 & 15,9 & 15,8 & 15,4 & 17,8 & 21,2 & 20,6 & $17,7 \pm 2,26$ \\
\hline Fibrinog & pré & 2,24 & 3,60 & 2,22 & 2,68 & 2,22 & 2,50 & 4,50 & 2,67 & $3,16 \pm 1,57$ \\
\hline Vr-1,8-3,5g/dl & pós & 2,06 & 3,40 & 2,06 & 2,00 & 2,03 & 2,39 & 2,46 & 1,88 & $2,28 \pm 0,46$ \\
\hline ATIII & pré & 16,8 & 19,3 & 21,9 & 23,9 & 25,5 & 25,9 & 24,5 & 22,0 & $21,7 \pm 3,09$ \\
\hline Vr-22-39mg/dl & pós & 16,5 & 19,2 & 20,1 & 21,2 & 24,8 & 22,6 & 18,7 & 17,0 & $20,7 \pm 2,93$ \\
\hline TLE & pré & 150 & 160 & 160 & 160 & 160 & 160 & 160 & 160 & $158,7 \pm 3,30$ \\
\hline Vr-120-300min & pós & 150 & 160 & 160 & 60 & 160 & 160 & 160 & 160 & $146,2 \pm 32,7$ \\
\hline$D D D i^{*}$ & $\begin{array}{l}\text { pré } \\
\text { pós }\end{array}$ & $\begin{array}{l}4000 \\
4000\end{array}$ & $\begin{array}{l}<500 \\
2000\end{array}$ & $\begin{array}{c}<500 \\
500-1000\end{array}$ & $\begin{array}{l}1000 \\
1000\end{array}$ & $\begin{array}{l}500-1000 \\
500-1000\end{array}$ & $\begin{array}{l}<500 \\
<500\end{array}$ & $\begin{array}{l}<500 \\
<500\end{array}$ & $\begin{array}{c}500-1000 \\
<500\end{array}$ & \\
\hline
\end{tabular}

${ }^{*}$ Negativo $<500 \mathrm{ng} / \mathrm{ml}$; positivo $>500 \mathrm{ng} / \mathrm{m}$ 
Pontes J C D V, Matos M F C, Medeiros C G S, Silva A F, Duarte J J, Gardenal N, Viola M D Z - Estudo comparativo do emprego da aprotinina em baixas doses X placebo, durante a circulação extracorpórea. Rev Bras Cir Cardiovasc 2002; 17(1): 47-53.

\section{CONCLUSÕES}

A utilização da aprotinina, mesmo em baixa dose, foi eficaz na redução do sangramento $(p=$ $0,0017)$ em pacientes adultos submetidos à operação cardíaca com CEC.

A diminuição da concentração de fibrinogênio $(p=0,0166)$ foi menor no grupo tra- tado do que no grupo controle, enquanto que, para o tempo de lise da euglobulina (TLE) não houve diferença significativa; entretanto, o efeito da aprotinina inibindo a fibrinólise pode ser observado por menores níveis de dímero-D no grupo que recebeu a droga $(p=0,0065)$.

RBCCV 44205-571

Pontes J C D V, Matos M F C, Medeiros C G S, Silva A F, Duarte J J, Gardenal N, Viola M D Z - Comparative study of low-dose aprotinin x placebo during cardiopulmonary bypass. Rev Bras Cir Cardiovasc 2002; 17(1): 47-53.

ABSTRACT: Background: The use of aprotinin, a antifibrinolytic agent, has been shown to decrease damaging effects on cardiopulmonary bypass in fibrinolytic system, which may improve hemostasis. Objective: To study the effect of low dose aprotinin in patients undergoing extracorporeal circulation.

Method: Seventeen patients, underwent cardiopulmonary bypass to mitral valve replacement, was ramdomized in two groups: I (control) - 9 patients received placebo after anesthesia induction and each hour in the priming; II (aprotinin) - 8 patients received after anesthesia induction 30,000 KIU/ $\mathrm{kg}$ and $7.500 \mathrm{KIU} / \mathrm{kg}$ each hour in the priming during the perfusion. The blood loss was observed through the first 24 hours postoperatively. Arterial blood samples were taken after anesthesia induction and after administration of protamina in order to analyse: prothrombin activity (PA), partial thomboplastin time (PTT), thrombin time (TT), euglobulin lysis time (ELT) and to measure levels of fibrinogen ( $F)$, d-dimer (dD) and antithrombin III (ATIII).

Results: Mean postoperative bleeding at the 24th hours was $690.67 \pm 377$ in the control group and $248, .75 \pm 105$ in the aprotinin group $(p=0.0017)$. The results taken from the blood samples were shown above.

\begin{tabular}{lccc}
\hline Examination & Period & Group I & Group II \\
AP & pre op & $81,2 \pm 13,3$ & $83,6+14,92$ \\
Vr-70-130Seg & post op & $54,5 \pm 11,0$ & $61,3+12,25$ \\
TIPA & pre & $34,1 \pm 8,1$ & $34,9+6,39$ \\
Vr-22-35Seg & post op & $40,6 \pm 7,1$ & $44,9+8,06$ \\
TT & pre & $15,4 \pm 1,1$ & $15,9+2,38$ \\
Vr-14-21Seg & post op & $17,5 \pm 1,1$ & $17,7+2,26$ \\
Fibrinog & pre & $3,04 \pm 0,84$ & $3,16+1,57$ \\
Vr-1,8-3,5g/dl & post op & $1,90 \pm 0,36$ & $2,28+0,46$ \\
ATIII & pre & $21,8+2,9$ & $21,7+3,09$ \\
Vr-22-39mg/dl & post op & $19,1 \pm 2,9$ & $20,7+2,93$ \\
TLE & pre & $160 \pm 25,30$ & $158,7+3,30$ \\
Vr-120-300min & post op & $127 \pm 61,69$ & $146,2+32,7$ \\
\hline
\end{tabular}

Conclusion: It follows that aprotinin, in low dose, was able to inhibit fibrinolysis and reduced bleeding after cardiopulmonary bypass.

DESCRIPTORS: Aprotinin, therapeutic use. Extracorporeal circulation, adverse effects. 
Pontes J C D V, Matos M F C, Medeiros C G S, Silva A F, Duarte J J, Gardenal N, Viola M D Z - Estudo comparativo do emprego da aprotinina em baixas doses X placebo, durante a circulação extracorpórea. Rev Bras Cir Cardiovasc 2002; 17(1): 47-53.

\section{REFERÊNCIAS BIBLIOGRÁFICAS}

1 Horrow J C - Management of coagulopathy associated with cardiopulmonary bypass. In: Gravlee G P, Davis R F, Utley J R, eds. Cardiopulmonary bypass: principles and practice. Williams \& Wilkins, Baltimore, 1993.

2 Ellison $\mathrm{N}$ \& Jobes $\mathrm{D}$ - Hemostasis during cardiopulmonary bypass. In: Tinker $\mathrm{J} \mathrm{H}$, ed. Cardiopulmonary bypass: current concepts and controversies. W. B. Saunders Co., Philadelphia, 1989.

3 Kucuk O, Kwaan H C, Frrederickson J, Wade L, Green D - Increased fibrinolytic activity in patients undergoing cardiopulmonary bypass operation. $A m \mathrm{~J}$ Hematol 1986; 23: 223-9.

4 Bidstrup B P - Blood conservation in cardiac surgery: can drugs help? Perfusion 1988; 3: 171-7.

5 Tice D A, Woeth M H, Clauss R H, Reed G E - The inhibition by trasylol of fibrinolytic activity associated with cardiovascular operations. Surg Gynecol Obstet 1964; 119: 71-4.

6 Van Oeveren W, Jansen N J G, Bidstrup B P et al. - Effects of aprotinin on haemostatic mechanism in cardiopulmonary bypass. Ann Thorac Surg 1987; 44: 640-5.

7 Royston D, Bidstrup B P, Taylor K M, Sapsfort R N Effect of aprotinin on need for blood transfusion after repeat open-heart surgery. Lancet 1987; 2(8571): 1289-91.

8 Bidstrup B P, Royston D, Sapsfort R N, Taylor K M Reduction in blood loss and blood use after cardiopulmonary bypass with high dose aprotinin (Trasylol). J Thorac Cardiovasc Surg 1989; 97: 364-72.

9 Lemnier J H, Stanford W, Bonney S L et al. - Aprotinin for coronary bypass operations: efficacy, safety, and influence on early saphenous vein graft patency. A multicenter, randomized, double-blind, placebocontrolled study. J Thorac Cardiovasc Surg 1994; 107: 543-53.

10 Goldstein D J, DeRosa C M, Mongero L B et al. - Safety and efficacy of aprotinin under conditions of deep hypothermia and circulatory arrest. J Thorac Cardiovasc Surg 1995; 110: 1615-22.
11 Colman R W - Hemostatic complications of cardiopulmonary bypass. Am J Hematol 1995; 48: 267-72.

12 Herynkopf F, Luchese F, Pereira E, Kalil R, Prates P, Nesralla I A - Aprotinin in children undergoing correction of congenital heart defects: a double blind pilot study. J Thorac Cardiovasc Surg 1994; 108: 517-21.

13 Havel M, Grabenwoger F, Schneider J et al. Aprotinin does not decrease early graft patency after coronary artery bypass grafting despite reducing postoperative bleeding and use of donated blood. J Thorac Cardiovasc Surg 1994; 107: $807-10$.

14 Liu B, Tengborn L, Larson G et al. - Half-dose aprotinin preserves hemostatic function in patients undergoing bypass operations. Ann Thorac Surg 1995; 59: 1534-40.

15 Mastroroberto P, Chello M, Zofrea S, Marchese A R Supressed fibrinolysis after administration of low-dose aprotinin: reduced level of plasmin-alpha2-plasmin inhibitor complexes and postoperative blood loss. Eur J Cardio Thorac Surg 1995; 9: 143-5.

16 Dietrich W, Spannagel M, Jochum M et al. - Influence of high-dose aprotinin treatment on blood loss and coagulation patterns in patients undergoing myocardial revascularization. Anesthesiology 1990; 73: 1119-26.

17 Cosgrove D M 3rd, Heric B, Lytle B W et al. - Aprotinin therapy for reoperative myocardial revascularization: a placebo-controlled study. Ann Thorac Surg 1992; 54: $1031-8$.

18 Liu B, Belboul A, Radberg G et al. - Effect of reduced aprotinin dosage on blood loss and use of blood products in patients undergoing cardiopulmonary bypass. Scand J Thorac Cardiovasc Surg 1993; 27: 149-55.

19 Hardy J F, Desroches J, Belisle S, Perrault J, Carrier $M$, Robitaille D - Low-dose aprotinin infusion is not clinically useful to reduce bleeding and transfusion of homologous blood products in highrisk cardiac surgical patients. Can J Anaesth 1993; 40: 625-31. 